\title{
Reguladores de integridad endotelial como posibles predictores de la gravedad en casos de dengue
}

\author{
Claudia Lucía Figueroa', Margarita Gélvez² ${ }^{2}$ Jürg Niederbacher ${ }^{3}$ \\ 1 Grupo GERMINA, Departamento de Medicina Interna, Escuela de Medicina, Facultad de Salud, Universidad \\ Industrial de Santander, Bucaramanga, Colombia \\ 2 Centro de Investigaciones Epidemiológicas, Facultad de Salud, Universidad Industrial de Santander, \\ Bucaramanga, Colombia \\ 3 Departamento de Pediatría, Escuela de Medicina, Facultad de Salud, Universidad Industrial de Santander, \\ Bucaramanga, Colombia
}

Introducción. El dengue es una de las enfermedades trasmitidas por mosquitos de mayor impacto en el mundo. La evolución clínica de la enfermedad suele ser impredecible, por lo cual su adecuado manejo en las fases tempranas podría incidir en la mejoría del paciente.

Objetivo. Evaluar los niveles séricos de algunos reguladores endoteliales (VEGF, sICAM-1, endoglina soluble, Ang-1 y Ang-2) como marcadores de predicción de la gravedad del dengue.

Materiales y métodos. Se hizo un estudio de casos y controles anidado en una cohorte. En la fase temprana, los niveles de los reguladores endoteliales se midieron con ELISA. La relación entre las variables clínicas y los reguladores se analizó mediante regresión logística utilizando como variable de salida la gravedad del dengue. Con base en la relación entre las variables de interés y el resultado, se estableció un posible modelo predictor de la gravedad empleando la mejor área bajo la curva (ROC).

Resultados. La mediana de la edad fue de 24 años. Los casos graves se asociaron con niveles séricos de Ang-2 a partir de un punto de corte mayor o igual a $1.490 \mathrm{pg} / \mathrm{ml}$, (Odds ratio, OR=3,1 p=0,015). Los niveles séricos de Ang-2, así como un área de 0,73 bajo la curva ROC, contribuyeron al modelo de predicción de la gravedad, conjuntamente con las variables de exantema, trastorno de conciencia y dolor abdominal, con OR de 3,2 (IC $\left.\mathrm{IS}_{5 \%} 1,16-8,9 ; \mathrm{p}=0,024\right)$.

Conclusión. El regulador endotelial Ang-2 podría ser un predictor de la gravedad en el dengue.

Palabras clave: endotelio, dengue, dengue grave, riesgo, permeabilidad capilar, predicción. doi: http://dx.doi.org/10.7705/biomedica.v36i0.2878

\section{Regulators of endothelial integrity as severity predictors in dengue}

Introduction: Dengue is currently among the mosquito-borne diseases of greatest global impact. The clinical course of the disease can be unpredictable, so proper handling in its early stages is critical to ensure optimal outcomes.

Objective: To evaluate serum regulators of endothelial integrity (VEGF, sICAM-1, sEndoglina, Ang-1, and Ang-2) as predictive markers of dengue severity.

Materials and methods: We conducted a case-control study nested in an appropriate cohort. Endothelial regulator levels were first measured by ELISA, after which analysis was performed using logistic regression of clinical and regulatory variables, with severity as an output variable. A possible severity prediction model, based on the variables of interest and output, was defined using the best area under the ROC curve.

Results: The median subject age was 24 years. Severe cases were associated with Ang-2 serum levels of $\geq 1,490 \mathrm{ng} / \mathrm{ml}(\mathrm{OR}=3.1 ; \mathrm{p}=0.015)$. Serum levels of Ang-2 $(\geq 1,490 \mathrm{ng} / \mathrm{ml})$ contributed to the severity prediction model, as did a 0.73 area under the ROC curve, together with the variables rash, impaired consciousness and abdominal pain, with an OR of 3.2 ( $\mathrm{Cl}$ 95\%: 1.16 to 8.9; $\mathrm{p}=0.024)$.

Conclusion: The endothelial regulator Ang-2 could be a predictor of severity in dengue.

Key words: Endothelium, dengue, severe dengue, risk, capillary permeability, forecasting. doi: http://dx.doi.org/10.7705/biomedica.v36i0.2878

\footnotetext{
Contribución de los autores:

Jürg Niederbacher y Margarita Gélvez: recolección de datos

Margarita Gélvez: análisis de las pruebas de laboratorio

Claudia Lucía Figueroa: análisis estadístico

Todos los autores participaron en el diseño de la propuesta y en la escritura del manuscrito.
} 
El dengue es actualmente una de las enfermedades transmitidas por mosquitos de mayor interés en salud pública, debido a su incidencia y a la expansión del arbovirus hacia nuevas regiones del trópico y de las áreas subtropicales, por lo que hoy más de $40 \%$ de la población mundial está en riesgo de contraer la enfermedad (1). La prevención y el control del dengue dependen exclusivamente de las medidas eficaces de lucha antivectorial (1). En Colombia, $75 \%$ del territorio nacional está situado por debajo de los 1.800 metros y tiene condiciones de temperatura, humedad relativa y pluviosidad adecuadas para el vector $y$, además, se registra transmisión endémica urbana del dengue (2). En el 2013, los casos aumentaron más de $100 \%$ con relación al año anterior (de 43.000 casos a más de 100.000 casos), así como la mortalidad en los casos graves de la enfermedad (4,7\%) (3).

Dado que no existe un tratamiento antiviral específico, el suministro oportuno de grandes volúmenes de líquidos endovenosos es la única intervención que reduce la gravedad y evita los casos fatales. No obstante, la selección clínica de aquellos pacientes a quienes se les debe ofrecer este tratamiento es difícil, puesto que en los primeros días de la enfermedad aquellos que evolucionan hacia la gravedad presentan los mismos síntomas de quienes no lo hacen.

Debido a que el choque por dengue produce una disminución acentuada de la resistencia vascular, probablemente relacionada con la pérdida de la integridad capilar y la consecuente extravasación de líquidos, se asume que las formas graves del dengue deben obedecer a un proceso de disfunción endotelial $(4,5)$. Este mismo efecto se produce en otros procesos como la inflamación grave o la malaria cerebral, en cuyo desarrollo se observa que la disfunción endotelial desempeña un papel importante (6).

En los casos graves de dengue se ha observado el comportamiento de varios reguladores endoteliales (7), entre los cuales se cuentan la angiopoyetina-1 (Ang-1) y la angiopoyetina-2 (Ang-2), que parecen ser la llave de la regulación de la integridad vascular (8). En pacientes con fiebre hemorrágica y

\footnotetext{
Correspondencia:

Claudia Lucía Figueroa, Carrera 32 № 29-31, Bucaramanga, Colombia

Teléfono: (577) 634 4000, extensión 3105

clalufi@uis.edu.co

Recibido: 06/06/15; aceptado: 22/03/16
}

choque por dengue se ha reportado la disminución de los niveles séricos de Ang-1 y el incremento en los niveles séricos de Ang-2 en la fase aguda en comparación con la fase de convalecencia y con individuos sanos de control. Al parecer, este desequilibrio contribuye a la fuga plasmática que se da en los casos de fiebre hemorrágica y choque por dengue $(9,10)$.

Otro protagonista en la regulación endotelial es el factor de crecimiento endotelial vascular (Vascular Endothelial Growth Factor, VEGF), el cual promueve el crecimiento, la proliferación y la migración de células endoteliales. El VEGF aumenta en pacientes con dengue grave (11). Asimismo, la acción conjunta del VEGF y la Ang-2 se ha asociado significativamente con la fuga plasmática (12).

Otro marcador importante de la angiogénesis es la endoglina, la cual se expresa de forma acentuada en las células endoteliales, en especial, en el tejido inflamado. La forma soluble de la endoglina es liberada en la membrana celular y entra en circulación, probablemente por ruptura, mediante las metaloproteinasas de la matriz, causando disfunción endotelial. En un estudio sobre el uso de la endoglina soluble como predictor de la malaria, se encontró que los niveles superiores a $12 \mathrm{pg} / \mathrm{ml}$ se presentaban con mayor frecuencia en la malaria grave $(46,5 \%)$ y en la malaria complicada $(85,7 \%)$ que en otras condiciones causantes de fiebre (0\%), así como en los individuos sanos de control (0\%) (6).

Las moléculas de adhesión intercelular (Intercellular Adhesion Molecule, ICAM-1) se expresan en muchos tipos de células, incluidos los leucocitos y las células endoteliales, y su forma soluble (sICAM-1) es la que se detecta en suero, por lo cual el hallazgo de niveles altos de esta forma soluble se ha asociado a la presencia de enfermedades cardiovasculares, alteraciones autoinmunitarios y cáncer. En muchos estudios se han correlacionado los niveles de sICAM-1 con la gravedad de estas enfermedades (13). En 2006, Khongphatthanayothin, et al., determinaron los niveles de sICAM-1 en pacientes con infección por el virus del dengue y encontraron que, en la etapa aguda de la enfermedad, dichos niveles eran significativamente más altos que en el seguimiento $(p=0,001)$; sin embargo, al compararlos con los del grupo sano, no se diferenciaron $(p=0,12)$ (14). En un estudio reciente se evaluaron varios reguladores endoteliales y se encontró que los 
niveles séricos de sICAM-1 y de la endoglina soluble fueron más elevados en los pacientes que posteriormente desarrollaron fiebre hemorrágica y choque por dengue, comparados con los pacientes con síndrome febril causado por Leptospira spp., lo cual sugiere que la activación endotelial podría ser una vía importante del desarrollo del dengue (7).

Otro aspecto importante de este estudio fue la evaluación de otros biomarcadores como posibles reguladores de la predicción temprana de la gravedad en casos de infección por el virus del dengue, entre ellos, los siguientes: Tie-1, Tie-2, Ang-like 3, Ang-like 4, PCR, IP10, IL-18, VEGF, PCR, Ang-1, Ang-2, IL-10, sVEGR-2/KDR, sFIt-1, factor D, C5a y CHI 3L1.

El comportamiento de estos biomarcadores ha avivado el interés por encontrar otros predictores de la gravedad $(7,15)$, contexto en el cual se inscribieron los objetivos de la presente investigación de caracterizar y precisar el comportamiento temprano de las moléculas VEGF, sICAM-1, endoglina soluble, Ang-1 y Ang-2 en nuestra población, además de establecer en qué medida dichos reguladores contribuyen en la predicción clínica de la gravedad de la enfermedad.

\section{Materiales y métodos}

Se hizo un estudio de casos y controles anidado en una cohorte de pacientes hospitalizados 0 ambulatorios de seis meses de edad en adelante con síndrome febril agudo de más de 48 horas y menos de 96 horas de evolución, sin foco aparente después del examen físico, con recuento plaquetario de más de 50.000 por $\mathrm{mm}^{3}$ y con al menos dos de las siguientes manifestaciones: cefalea, dolor retroocular, mialgias, artralgias, exantema, manifestaciones hemorrágicas menores y leucopenia $\left(<5.000 / \mathrm{mm}^{3}\right)$, según la definición operativa de caso sospechoso de dengue de la Organización Mundial de la Salud (OMS) (16). La infección por el virus del dengue se confirmó mediante alguna de las siguientes pruebas: seroconversión (ELISA IgM en muestra negativa de pacientes en fase aguda y en muestra positiva de los convalecientes), cuadruplicación de títulos de anticuerpos en muestras pareadas, aislamiento viral y reacción en cadena de la polimerasa en tiempo real (RT-PCR).

El caso se definió como el de un paciente que presentara uno o más de los siguientes hallazgos clínicos: dengue grave o choque por dengue según la definición de 1997 de la OMS (16), hipotensión y sangrado mayor.
El primer grupo de control incluyó pacientes que presentaran uno o más de los siguientes hallazgos clínicos: dengue no grave según la definición de la OMS de 1997 (16), sin hipotensión y sin sangrado mayor. El segundo grupo de control incluyó a pacientes con síndrome febril agudo inespecífico con menos de 96 horas de evolución y diagnóstico de leptospirosis confirmado mediante ensayo de inmunofluorescencia indirecta (IFI).

Para el cálculo del tamaño se tuvieron en cuenta los valores de los niveles de VEGF, sICAM-1, endoglina soluble, Ang-1 y Ang-2 encontrados en otra enfermedad febril de potencial curso grave (malaria), pero teniendo en cuenta las diferencias entre malaria cerebral $(p<0,001)$ y malaria no complicada $(p<0,001)(17)$. Con un poder de $90 \%$ y un error alfa de $5 \%$, en una relación entre caso y controles uno y dos de 1:1:1, se estimó que se requerían 30 pacientes con dengue grave, 30 pacientes con dengue no grave y 30 pacientes con leptospirosis.

Tanto los casos como los controles se agruparon por edad y sexo, y se seleccionaron aleatoriamente. Los niveles de VEGF, sICAM-1, endoglina soluble, Ang-1 y Ang-2 se midieron con estuches comerciales de ELISA (Quantikine Human, R\&D Systems), siguiendo las instrucciones del fabricante. Las muestras de análisis las constituyeron los sueros (sin anticoagulante) de casos y controles almacenados a $-80^{\circ} \mathrm{C}$ que nunca hubieran sufrido ciclos de descongelamiento.

Se compararon los niveles de los marcadores estudiados y las variables sociodemográficas y clínicas de casos y controles, en busca de diferencias entre los grupos mediante un análisis bivariado con la prueba de t de Student o de MannWhitney según la normalidad en la distribución de las variables continuas $y$, para las variables categóricas, la prueba de ji al cuadrado.

Se hizo un análisis multivariado mediante el modelo de regresión logística utilizando como variable de salida la gravedad del dengue y, como variables independientes, los niveles de VEGF, sICAM-1, endoglina soluble, Ang-1 y Ang-2, además de las variables clínicas que tuvieron significación estadística en el análisis bivariado y que modificaron en más de $10 \%$ los valores estimados en el modelo. Posteriormente, aquellas variables que tuvieron significación estadística en el análisis multivariado se incluyeron en la escala de predicción de la gravedad estimando el área 
bajo la curva ROC, con el fin de determinar su exactitud para la predicción de la gravedad, con o sin los reguladores inflamatorios.

Para el procesamiento y el análisis de los datos se empleó el programa estadístico Stata ${ }^{\circledR}$, versión 10,0 (Stata Corp).

Este fue un estudio con un riesgo menor al establecido como mínimo en la Resolución 008430 de 1993 expedida por el Ministerio de Salud (18). En el momento de la captación de pacientes y de la toma de muestras, se solicitó el consentimiento informado a ellos, o a sus padres en el caso de los menores de 18 años, así como autorización para almacenar los sueros y emplearlos en estudios posteriores.

\section{Resultados}

Los casos de fiebre por dengue y dengue grave se presentaron en su mayoría en mujeres (60\%) y, la mayoría de los casos de leptospirosis $(87 \%)$, en hombres. Las medianas de edad en los casos de fiebre por dengue fueron de 24 años, con un rango intercuartílico $(\mathrm{RIC})$ de 10 a 49, y en los de dengue grave, de 31,8 años, con un RIC de 8 a 46 , en tanto que en los de leptospirosis fue de 23 años, con un RIC de 7 a 81. La mayoría de los pacientes (30\%) provenía de Bucaramanga, uno de los cinco municipios estudiados.

Los niveles en suero de VEGF, sICAM-1, endoglina soluble, Ang-1 y Ang-2 en los pacientes con leptospirosis, fiebre por dengue y dengue grave, presentaron un comportamiento muy homogéneo, con excepción de los niveles de Ang-2 en los casos de dengue grave (cuadro 1). Con base en el análisis de las cifras crudas comparadas con las medianas, se sugirió que el Ang-2 podría tener un comportamiento diferente $(p=0,096)$ (cuadro 1), con una mayor tendencia a concentrar los valores (figura 1).

Al hacer la regresión logística entre la variable de tipo de caso (dengue grave o no) y la variable de la Ang-2, se obtuvo una OR de $1,09\left(\mathrm{IC}_{95 \%} 0,9-\right.$

Cuadro 1. Niveles de los reguladores de integridad endotelial en los casos y los controles

\begin{tabular}{lrrrr}
\hline Biomarcador & $\begin{array}{c}\text { Fiebre por } \\
\text { dengue }\end{array}$ & $\begin{array}{c}\text { Dengue } \\
\text { grave }\end{array}$ & $\begin{array}{c}\text { Leptospira } \\
\text { spp. }\end{array}$ & p \\
\hline sICAM* $^{*}$ & 170,245 & 19,303 & 19,703 & 0,66 \\
Angio1* $_{\text {Angio2 }}^{716,645}$ & 9,960 & 986,146 & 0,32 \\
VEGF* $^{*}$ & 10,267 & 13,825 & 1,222 & 0,09 \\
sEndoglobina* $^{*}$ & 159,754 & 209,632 & 229,978 & 0,66 \\
\hline
\end{tabular}

*Mediana. Niveles expresados en pg/ml. p: significación estadística
$2,3 ; p=0,095)$. Se hizo, entonces, la regresión nuevamente, pero categorizando los valores de este biomarcador (Angio-2cat) hasta encontrar que en los casos con niveles séricos mayores de 1.490 $\mathrm{pg} / \mathrm{ml}$ la probabilidad de dengue grave aumentaba tres veces, con una OR de $3,16\left(\mathrm{IC}_{95 \%}, 1,2-5,13\right)$ y una significación estadística adecuada ( $p=0,015)$, aunque con una capacidad de predicción o área bajo la curva ROC de 0,62 (figura 2). Los demás reguladores no mostraron relación con el resultado de dengue grave en la población estudiada.

Al comparar el comportamiento de las variables clínicas (cuadro 2), solo algunas de las variables de riesgo de gravedad (hemorragia gastrointestinal, hemorragia genitourinaria, náuseas, vómito, hemorragia nasal, hepatomegalia, exantema, petequias, dolor abdominal, prurito, enrojecimiento de los ojos, o trastorno de conciencia), se relacionaron individualmente con el resultado en la evaluación paso a paso. Sin embargo, solo una de ellas tuvo una adecuada significación estadística

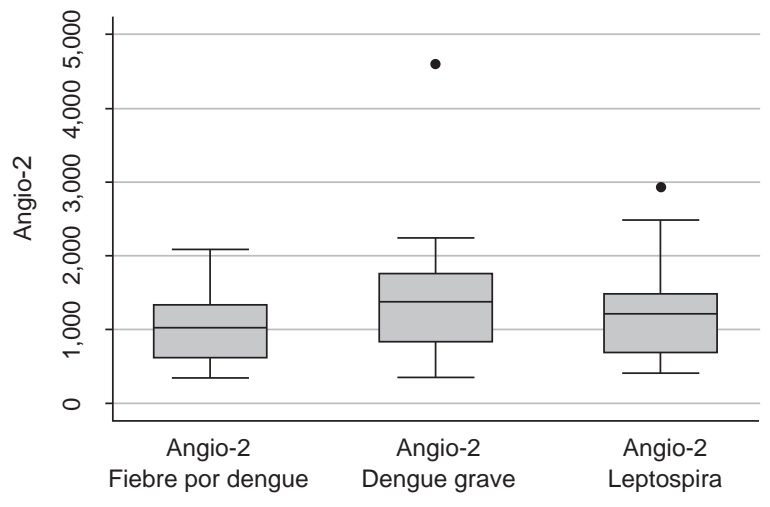

Figura 1. Distribución de Ang-2 según el tipo de caso o control

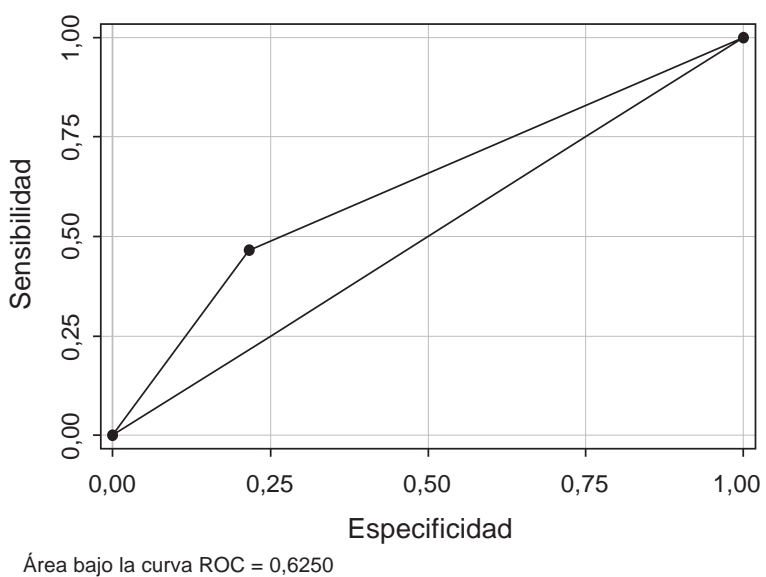

Figura 2. Curva ROC en el modelo bivariado (tipo-caso/Ang2cat>1.490 ng/ml 
Cuadro 2. Variables clínicas

\begin{tabular}{|c|c|c|c|c|c|c|c|c|}
\hline \multirow{3}{*}{$\begin{array}{l}\text { Variable clínica } \\
\text { Cefalea }\end{array}$} & \multicolumn{7}{|c|}{ Clasificación clínica n (\%) $\left(\mathrm{IC}_{95 \%}\right)$} & \multirow{3}{*}{$\begin{array}{c}\begin{array}{c}\text { Significación } \\
\text { estadística }\end{array} \\
\mathbf{p} \\
0,35\end{array}$} \\
\hline & \multicolumn{3}{|c|}{ Fiebre por dengue } & \multicolumn{2}{|c|}{ Dengue grave } & \multicolumn{2}{|c|}{ Leptospira spp. } & \\
\hline & 30 & $(100)$ & & $28(93)$ & $(77-99)$ & $29(96)$ & $(82-99)$ & \\
\hline Dolor retroocular & 23 & $(76)$ & $(57-90)$ & $24(80)$ & $(61-92)$ & $20(66)$ & $(47-82)$ & 0,46 \\
\hline Astenia & 20 & $(66)$ & $(47-82)$ & $26(86)$ & $(69-96)$ & $23(76)$ & $(73-97)$ & 0,18 \\
\hline Dolor muscular & 29 & (96) & $(61-92)$ & $28(93)$ & $(77-99)$ & $27(90)$ & $(73-97)$ & 0,54 \\
\hline Dolor articular & 26 & $(86)$ & $(69-96)$ & $22(73)$ & $(54-87)$ & $22(73)$ & $(54-87)$ & 0,35 \\
\hline Escalofrío & 29 & (96) & $(61-92)$ & $29(96)$ & $(61-92)$ & $27(90)$ & $(73-97)$ & 0,42 \\
\hline Disminución del apetito & 23 & (76) & $(73-97)$ & $22(73)$ & $(54-87)$ & $24(96)$ & $(82-99)$ & 0,03 \\
\hline Dolor de garganta & 9 & (30) & $(14-49)$ & $13(43)$ & $(25-62)$ & $18(60)$ & $(40-77)$ & 0,06 \\
\hline Tos & 10 & (33) & $(17-52)$ & $13(43)$ & $(25-62)$ & $15(50)$ & $(31-68)$ & 0,42 \\
\hline Secreción u obstrucción nasal & 7 & (23) & $(9-42)$ & $13(43)$ & $(25-62)$ & $15(50)$ & $(31-68)$ & 0,08 \\
\hline Ardor de la piel & 5 & (16) & (5-34) & $9(30)$ & $(14-49)$ & $5(16)$ & $(5-34)$ & 0,34 \\
\hline Exantema & 11 & (36) & $(19-56)$ & $16(53)$ & $(34-71)$ & $8(26)$ & $(12-45)$ & 0,11 \\
\hline Prurito corporal & 15 & (50) & $(31-68)$ & $10(33)$ & $(17-52)$ & $6(20)$ & $(0,7-38)$ & 0,05 \\
\hline Vómito & 11 & (36) & $(19-56)$ & $11(36)$ & $(19-56)$ & $12(40)$ & $(22-59)$ & 0,95 \\
\hline Diarrea & 14 & $(46)$ & $(28-65)$ & $9(30)$ & $(14-49)$ & $9(30)$ & $(14-49)$ & 0,14 \\
\hline Dolor abdominal & 20 & $(66)$ & $(47-82)$ & $20(66)$ & $(47-82)$ & $16(53)$ & $(34-71)$ & 0,46 \\
\hline Visión borrosa & 8 & (26) & $(12-45)$ & $17(56)$ & $(37-74)$ & $10(33)$ & $(17-52)$ & 0,04 \\
\hline Mareo & 23 & (76) & $(57-90)$ & $23(76)$ & $(57-90)$ & $24(80)$ & $(61-92)$ & 0,93 \\
\hline Visión con luces o estrellas & 4 & $(13)$ & $(3-30)$ & $12(40)$ & $(22-59)$ & $13(43)$ & $(25-62)$ & 0,02 \\
\hline Convulsión & 0 & & & 0 & & 1 & & 0,36 \\
\hline Somnolencia & 19 & $(63)$ & $(43-80)$ & $21(70)$ & $(50-85)$ & $19(63)$ & $(43-80)$ & 0,81 \\
\hline Hemorragia gingival espontánea & 0 & & & 0 & & 1 & & 0,36 \\
\hline Hemorragia gingival no espontánea & 5 & $(16)$ & $(5-34)$ & $5(16)$ & (5-34) & $2(6)$ & $(0,08-22)$ & 0,42 \\
\hline Hemorragia nasal espontánea & 0 & & & $1(3,3)$ & $(0,008-17)$ & $1(3,3)$ & $(0,008-17)$ & 0,66 \\
\hline Hemorragia nasal no espontánea & 1 & $(3,3)$ & $(0,008-17)$ & 0 & & $3(10)$ & $(0,2-26)$ & 0,16 \\
\hline Vómito de sangre oscura & 1 & $(3,3)$ & $(0,008-17)$ & 0 & & 0 & & 0,36 \\
\hline Frecuencia respiratoria >24 & 3 & (10) & $(0,2-26)$ & $4(13)$ & $(3-30)$ & $4(13)$ & $(3-30)$ & 0,92 \\
\hline Temperatura $<37^{\circ} \mathrm{C}$ & 21 & (70) & $(50-85)$ & $15(50)$ & $(31-68)$ & $24(80)$ & $(61-92)$ & 0,04 \\
\hline Deshidratación & 16 & (53) & $(34-71)$ & $17(56)$ & $(37-74)$ & $20(66)$ & $(47,82)$ & 0,55 \\
\hline Alteración de la conciencia & 5 & $(16)$ & $(5-34)$ & $6(20)$ & $(0,7-38)$ & $1(3,3)$ & $(0,008-17)$ & 0,13 \\
\hline Eritema facial & 12 & $(40)$ & $(22-59)$ & $15(50)$ & $(31-68)$ & $8(26)$ & $(12-45)$ & 0,17 \\
\hline Enrojecimiento de los ojos & 10 & (33) & $(17-52)$ & $12(40)$ & (22-59) & $9(30)$ & $(14-49)$ & 0,77 \\
\hline Presión arterial media $<70 \mathrm{~mm} \mathrm{Hg}$ & 1 & $(3,3)$ & $(0,008-17)$ & $2(6)$ & $(0,08-22)$ & 0 & & 0,35 \\
\hline Presión del pulso $<20 \mathrm{~mm} \mathrm{Hg}$ & 0 & & & 0 & & 0 & & \\
\hline Hipotensión ortostática & 2 & (6) & $(0,08-22)$ & $6(20)$ & $(0,7-38)$ & $6(20)$ & $(0,7-38)$ & 0,26 \\
\hline Edema palpebral & 6 & $(20)$ & $(0,7-38)$ & $1(3,3)$ & $(0,008-17)$ & $1(3,3)$ & $(0,008-17)$ & 0,82 \\
\hline Derrame pericárdico & 0 & & & 0 & & 0 & & \\
\hline Fiebre & 26 & $(86)$ & $(69-96)$ & $23(76)$ & $(57-90)$ & $22(73)$ & $(54-87)$ & 0,70 \\
\hline Derrame pleural & 0 & & & 0 & & 0 & & \\
\hline Hepatomegalia & 0 & & & $5(16)$ & $(5-34)$ & $4(13)$ & $(3-30)$ & 0,75 \\
\hline Dolor abdominal & 11 & $(36)$ & $(19-56)$ & $16(53)$ & $(34-71)$ & $10(33)$ & $(17-52)$ & 0,24 \\
\hline Abdomen en tabla & 0 & & & 0 & & 0 & & \\
\hline Prueba de torniquete positiva & 14 & $(46)$ & $(28-65)$ & $15(50)$ & $(31-68)$ & $11(36)$ & $(19-56)$ & 0,56 \\
\hline Petequias & 2 & (6) & $(0,08-22)$ & $5(16)$ & $(5-34)$ & $1(3,3)$ & $(0,008-17)$ & 0,16 \\
\hline
\end{tabular}

n: número; p: significación estadística

en el modelo de regresión logística. Al asociarse con el exantema, la Ang-2 (mayor de 1.490 pg/ $\mathrm{ml}$ ) mantuvo la misma probabilidad de predecir el dengue grave $(\mathrm{OR}=3,3)$, con una $\mathrm{OR}$ estimada de 4,24 para que el exantema aumentara dicha probabilidad ( $\mathrm{IC}_{95 \%} 1,61-11 ; \mathrm{p}=0,03$ ) (cuadro 3), al presentar una mejor capacidad de predicción (área debajo de la curva ROC de 0,72 ) (figura 3), con una sensibilidad de $30 \%$, una especificidad de $95 \%$, un valor predictivo positivo de $75 \%$ y uno negativo de $73 \%$. Las demás variables clínicas, a pesar de presentar riesgos estimados (OR) mayores de 2,0, tuvieron intervalos de confianza (95\%) muy amplios y una significación estadística por encima de 0,05 (cuadro 4). Además, se comportaron de manera muy similar entre los grupos de pacientes analizados, con pocas diferencias entre los casos y los controles $(p>0,05)$. 
Cuadro 3. Variable clínica predictora en el modelo logístico

\begin{tabular}{lccccc}
\hline $\begin{array}{l}\text { Dengue } \\
\text { grave }\end{array}$ & OR & $\begin{array}{c}\text { Error } \\
\text { estándar }\end{array}$ & $\mathbf{z}$ & $\mathbf{p}$ & $\mathbf{I C}_{95 \%}$ \\
\hline Ang-2cat & 3,3 & 1,74 & 2,3 & 0,018 & $1,17-8,12$ \\
Exantema & 4,24 & 2,09 & 2,93 & 0,003 & $1,61-11,1$ \\
\hline
\end{tabular}

OR: razón de momios; p: significación estadística; $\mathrm{IC}_{95 \%}$ : intervalo de confianza de $95 \%$

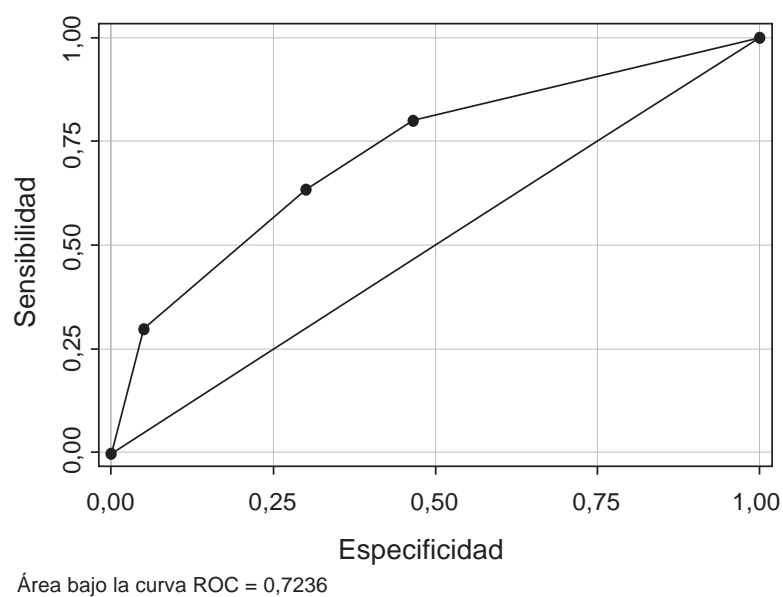

Figura 3. Curva ROC en el modelo multivariado (tipo-caso/ Ang-2cat/exantema)

A pesar de contar con una sola variable clínica y un solo biomarcador, en el modelo multivariado se observó una mejor capacidad de predicción del dengue grave, lo cual se reflejó en el cambio de 0,62 a 0,72 en el área bajo la curva (figuras 2 y 3).

\section{Discusión}

En la última década se han publicado varios estudios sobre el comportamiento de los casos de dengue grave, en los cuales el antecedente clínico de exposición previa al virus del dengue y los signos de fuga plasmática han sido los predictores más aceptados $(19,20)$. Sin embargo, la detección oportuna de quién va a desarrollar la fuga plasmática y cómo tratarla sigue siendo un reto (21).

Se sabe que, en la reacción inmunitaria al virus del dengue, las células vasculares o hematopoyéticas son de vital importancia, y que su comportamiento es similar al de los reguladores inflamatorios y endoteliales que participan en la activación y posterior disfunción endotelial en los procesos autoinmunitarios e infecciosos similares al dengue $(15,22)$. En todos ellos, la fuga plasmática y la hemorragia constituyen la expresión fundamental de la gravedad de los casos y el blanco de la investigación en torno a herramientas para el tratamiento temprano $(7,11,23,24)$. Específicamente, en un estudio llevado a cabo en el área metropolitana de Bucaramanga por Villar, et al., se determinó la capacidad de algunos biomarcadores para diferenciar entre síndrome febril por dengue y leptospirosis (25). Entre los 19 reguladores evaluados, los niveles de Angptl3, CXCL10, sICAM-1, IL-18BP y endoglina soluble fueron diferentes en los pacientes con una y otra infección $(p=0,001)(25)$.

A pesar de que en el presente estudio, el regulador de activación endotelial Ang-2 se expresó con ligeras diferencias en los casos de dengue grave, fiebre por dengue y leptospirosis, siempre se ha comportado de forma diferente en los casos graves y en los que no lo son, y en los sujetos sanos y tanto en enfermedades infecciosas diferentes al dengue como en reacciones homeostáticas de inflamación y coagulación en pruebas de laboratorios $(4,26)$. Se ha observado que los reguladores Ang-1 y Ang-2 en infantes con infecciones graves por dengue tienen un comportamiento característico (disminución en la expresión de Ang-1 y elevación en la de Ang-2) (27).

Se sabe el papel que, conjuntamente con otros reguladores, desempeña la Ang 1 en la integridad vascular, y el hecho de que se almacena en los pericitos y músculo liso de las células endoteliales, así como dentro de las plaquetas (28). En cuanto a la Ang-2, se almacena en los cuerpos de WeibelPalade, de donde se libera para los procesos de desestabilización del endotelio por disrupción celular, antagonizando los efectos de la Ang-1 e incrementando la inflamación y la activación endotelial, con la consecuente pérdida de plasma y hemorragia $(15,28)$. Se ha observado que las dos son relevantes en los procesos que desembocan en la gravedad de los pacientes en estado crítico, sin que hasta la fecha se hayan dilucidado completamente los mecanismos de dicha actividad (29).

Cuadro 4. Modelado con otras variables clínicas

\begin{tabular}{lcccl}
\hline $\begin{array}{l}\text { Dengue } \\
\text { grave }\end{array}$ & OR & $\begin{array}{c}\text { Error } \\
\text { estándar }\end{array}$ & $\mathbf{p}$ & $\mathbf{I C}_{95 \%}$ \\
\hline Ang-2cat & 3,22 & 1,67 & 0,24 & $1,16-8,94$ \\
Exantema & 2,66 & 1,25 & 0,032 & $0,99-6,8$ \\
Alteración de la & 2,7 & 1,84 & 0,052 & $0,90-13,7$ \\
$\begin{array}{l}\text { conciencia } \\
\text { Dolor abdominal }\end{array}$ & 2,29 & 1,09 & 0,057 & $0,74-5,20$ \\
\hline
\end{tabular}

OR: razón de momios; p: significación estadística; $I_{95 \%}$ : intervalo de confianza de $95 \%$ 
En cuanto a los demás reguladores endoteliales, se ha observado que en poblaciones heterogéneas (modelos animales y pacientes con dengue grave), algunos de ellos se han correlacionado con manifestaciones vasculares e inflamatorias, lo cual podría ser un campo de desarrollo de tratamientos farmacológicos $(22,28)$.

El presente estudio presenta algunas limitaciones en el sentido de que se trató de un estudio de casos y controles retrospectivo, que no alcanzó el poder esperado, pues el tamaño de la muestra no fue lo suficientemente representativo como para extrapolar los resultados a todos los casos graves por edad, sexo, o signos clínicos, y para establecer o descartar más asociaciones entre estos y los reguladores endoteliales.

En conclusión, el aumento de la probabilidad del dengue complicado se relacionó con niveles elevados de Ang-2, habiendo establecido un punto de corte para la población estudiada de $1.490 \mathrm{pg} / \mathrm{ml}$, lo que permite proponer su expresión como predictora independiente de gravedad. Se comprobó, igualmente, la variabilidad de la condición clínica que precede a la gravedad, lo cual abre la puerta al desarrollo de nuevos estudios orientados a entender mejor el papel de los reguladores de la integridad endotelial en el desarrollo de la gravedad en el dengue.

\section{Agradecimientos}

Al Centro de Investigaciones Epidemiológicas de la Universidad Industrial de Santander, Red AEDES.

\section{Conflicto de intereses}

Los autores manifiestan que no tuvieron conflictos de intereses en la realización del estudio ni en la redacción del manuscrito.

\section{Financiación}

Este estudio fue financiado por la Universidad Industrial de Santander (convocatoria interna número 5681).

\section{Referencias}

1. World Health Organization. Dengue and severe dengue. Fecha de consulta: $1^{10}$ de marzo de 2013. Disponible en: http://www.who.int/mediacentre/factsheets/fs117/en

2. Bello SL. Comportamiento epidemiológico del dengue en Colombia, año 2011. Fecha de consulta: 1을 de diciembre de 2012. Disponible en: http://www.ins.gov.co/lineas-de-accion/ Subdireccion-Vigilancia/Informe\%20de\%20Evento\%20 Epidemiolgico/Dengue\%202011.pdf

3. Ministerio de Salud y Protección Social. Informe de actividades al Congreso 2012-2013. Bogotá: Ministerio de Salud y Protección Social; 2013. p. 76-7. Fecha de consulta:
$1^{0}$ de noviembre de 2013. Disponible en: https://www. minsalud.gov.co/Documentos\%20y\%20Publicaciones/ Informe\%20al\%20Congreso\%202012-2013.pdf

4. Basu A, Chaturvedi UC. Vascular endothelium: The battlefield of dengue viruses. FEMS Immunol Med Microbiol. 2008;53:287-99. http://dx.doi.org/10.1111/j.1574695X.2008.00420.x

5. Vervaeke $\mathbf{P}$, Vermeire $\mathbf{K}$, Liekens $\mathbf{S}$. Endothelial dysfunction in dengue virus pathology. Rev Med Virol. 2015;25:5067. http://dx.doi.org/10.1002/rmv.1818

6. Dietmann A, Helbok R, Lackner P, Fischer M, Reindl M, Lell B, et al. Endoglin in African children with Plasmodium falciparum malaria. A novel player in severe malaria pathogenesis? J Infect Dis. 2009;200:1842-8. http://dx.doi. org/10.1086/648476

7. Conroy AL, Gélvez M, Hawkes M, Rajwans N, Liles WC, Villar-Centeno LA, et al. Host biomarker distinguish dengue from leptospirosis in Colombian: A case-control study. BMC Infect Dis. 2014;14:35. http://dx.doi.org/10.1186/1471-233414-35

8. van Meurs $M$, Kümpers $P$, Ligtenberg $J J$, Meertens JH, Molema G, Zijlstra JG. Bench-to-bedside review: Angiopoietin signalling in critical illness - a future target? Crit Care. 2009;13:207. http://dx.doi.org/10.1186/cc7153

9. Michels M, van der Ven AJ, Djamiatun K, Fijnheer R, de Groot PG, Griffioen AW, et al. Imbalance of angiopoietin-1 and angiopoetin-2 in severe dengue and relationship with thrombocytopenia, endothelial activation, and vascular stability. Am J Trop Med Hyg. 2012;87:943-6. http://dx.doi. org/10.4269/ajtmh.2012.12-0020

10. Ong SP, Ng ML, Chu JJ. Differential regulation of angiopoietin 1 and angiopoietin 2 during dengue virus infection of human umbilical vein endothelial cells: Implications for endothelial hyperpermeability. Med Microbiol Immunol. 2013;202:43752. http://dx.doi.org/10.1007/s00430-013-0310-5

11. Srikiatkhachorn A, Ajariyakhajorn C, Endy TP, Kalayanarooj S, Libraty DH, Green S, et al. Virus-induced decline in soluble vascular endothelial growth receptor 2 is associated with plasma leakage in dengue hemorrhagic fever. J Virol. 2007;81:1592-600. http://dx.doi.org/10.1128/ JVI.01642-06

12. van de Weg CA, Pannuti CS, van den Ham HJ, de Araújo ES, Boas LS, Felix AC, et al. Serum angiopoietin-2 and soluble VEGF receptor 2 are surrogate markers for plasma leakage in patients with acute dengue virus infection. $J$ Clin Virol. 2014;60:328-35. http://dx.doi.org/10.1016/j.jcv. 2014.05.001

13. Lawson C, Wolf S. ICAM-1 signaling in endothelial cells. Pharmacol Rep. 2009;61:22-32.

14. Khongphatthanayothin A, Phumaphuti P, Thongchaiprasit K, Poovorawan Y. Serum levels of SICAM-1 and sE-selectin in patients with dengue virus infection. Jpn $\mathrm{J}$ Infect Dis. 2006;59:186-8.

15. Page AV, Liles WC. Biomarkers of endothelial activation/ dysfunction in infectious diseases. Virulence 2013;4:50716. http://dx.doi.org/10.4161/viru.24530

16. World Health Organization. Clinical diagnosis. En: Dengue haemorrhagic fever: Diagnosis, treatment, prevention and control. Second edition. Geneva: WHO; 1997. p. 12-23. 
Fecha de consulta: 11 de agosto de 2012. Disponible en: http://www.who.int/entity/csr/resources/publications/ dengue/012-23.pdf

17. Conroy AL1, Lafferty EI, Lovegrove FE, Krudsood S, Tangpukdee N, Liles WC, et al. Whole blood angiopoietin-1 and -2 levels discriminate cerebral and severe (non-cerebral) malaria from uncomplicated malaria. Malar J. 2009;8:295. http://dx.doi.org/10.1186/1475-2875-8-295

18. Ministerio de Salud. Resolución № 0008430 de 1993. República de Colombia, 1993. Fecha de consulta: 23 de septiembre de 2012. Disponible en: https://www.invima. gov.co/images/pdf/medicamentos/resoluciones/etica res_8430_1993.pdf

19. Conroy A, Gélvez M, Hawkes M, Rajwans N, Tran V, Liles WC, et al. Host biomarkers are associated with progression to dengue haemorrhagic fever: A nested case-control study. Int J Infect Dis. 2015;40:45-53. http://dx.doi.org/10.1016/j. ijid.2015.07.027

20. Simmons CP, Farrar JJ, Nguyen VV, Wills B. Dengue. N Engl J Med. 2012;366:1423-32. http://dx.doi.org/10.1056/ NEJMra1110265

21. Srikiatkhachorn A, Kelley JF. Endothelial cells in dengue hemorrhagic fever. Antiviral Res. 2014;109:160-70. http:// dx.doi.org/10.1016/j.antiviral.2014.07.005

22. Spiropoulou C, Srikiatkhchachorn A. The role of endothelial activation in dengue hemorrhagic and hantavirus pulmonary syndrome. Virulence. 201315;4:525-36. http://dx. doi.org/10.4161/viru.25569

23. Tseng CS, Lo HW, Teng HC, Lo WC, Ker CG. Elevated levels of plasma VEGF in patients with dengue hemorrhagic fever. FEMS Immunol Med Microbiol. 2005;43:99-102. http://dx.doi.org/10.1016/j.femsim.2004.10.004
24. Azizan A, Sweat J, Espino C, Gemmer J, Stark L, Kazanis D. Differential proinflammatory and angiogenesis-specific cytokine production in human pulmonary endothelial cells, HPMEC-ST1.6R infected with dengue-2 and dengue-3 virus. J Virol Methods. 2006;138:211-7. http://dx.doi.org/10. 1016/j.jviromet.2006.08.010

25. Villar-Centeno LA, Lozano-Parra A, Salgado-García D, Herrán $\mathrm{O}$. Alteraciones bioquímicas como marcadores predictores de gravedad en pacientes con fiebre por dengue. Biomédica. 2013;33:63-9. http://dx.doi.org/10.7705/ biomedica.v33i0.732

26. Fiedler U, Scharpfenecker M, Koidl S, Hegen A, Grunow V, Schmidt JM, et al. The Tie-2 ligand angiopoietin-2 is stored in and rapidly released upon stimulation from endothelial cell Weibel-Palade bodies. Blood. 2004;103: 4150-6. http://dx.doi.org/10.1182/blood-2003-10-3685

27. Giuliano JS Jr, Lahni PM, Harmon K, Wong HR, Doughty LA, Carcillo JA, et al. Admission angiopoietin levels in children with septic shock. Shock. 2007;28:650-4. http:// dx.doi.org/10.1097/shk.0b013e318123867b

28. Dalrymple NA, Mackow ER. Roles for endothelial cells in dengue virus infection. Adv Virol. 2012;2012:840654. http:// dx.doi.org/10.1155/2012/840654

29. Ricciuto DR, dos Santos CC, Hawkes M, ToltI LJ, Conroy AL, Rajwans N, et al. Angiopoietin-1 and angiopoietin-2 as clinically informative prognostic biomarkers of morbidity and mortality in severe sepsis. Crit Care Med. 2011;39:702-10. http://dx.doi.org/10.1097/CCM.0b013e318206d285. 